\title{
LOS ESTUDIOS SOCIOCULTURALES COMO ESTRATEGIA ACADÉMICA PARA LA COMPRENSIÓN DE LAS PROBLEMÁTICAS AMBIENTALES DEL TERRITORIO
}

\author{
Sociocultural Studies as an Academic Strategy for the \\ Understanding of Environmental Problems of the Territory
}

\author{
LEÓN FELIPE CUBILLOS-QUINTERO ${ }^{1}$ \\ ${ }^{1}$ Director de la Línea Estudios Socioculturales y Problemática Ambiental Grupo de \\ Investigación en Gestión y Educación Ambiental, Universidad Tecnológica de Pereira.
}

E-mail: leons@utp.edu.co

Recibido: 28 de Julio de 2015

Aceptado: 7 de Septiembre de 2015

\section{Resumen}

El presente artículo centra la discusión en la necesidad de asumir los estudios ambientales con el aporte indiscutible de los estudios socioculturales tanto desde la perspectiva filosófica, histórica, antropológica y política.El marco de entrada a la justificación de las ciencias ambientales es la comprensión integral de las problemáticas ambientales existentes en cada territorio. El artículo menciona las visiones reduccionistas generadas desde la academia y la práctica institucional, las ciencias naturales y las ciencias sociales, que obstaculizan la posibilidad de realizar estudios ambientales desde los antecedentes culturales.En primer lugar discute el origen y singularidad del término ambiente en autores latinoamericanos como Daniel Vidart y Augusto Ángel Maya; en segundo lugar, destaca diferentes modalidades de acceso a las ciencias ambientales: desde los estudios disciplinares hasta la interdisciplinariedad, el saber ambiental y la filosofía ambiental, la resistencia social y la "indisciplina", para culminar destacando las principales características y el papel educativo que deben cumplir la educación superior en los procesos de educación ambiental comprometidos con el ejercicio interdisciplinario de los estudios ambientales en los territorios nacionales, regionales y locales. Estas últimas consideraciones se presentan en el tercero y cuarto aparte.

Palabras claves: Estudios socioculturales, Problemática Ambiental, Territorio, Interdisciplina, Ciencias Ambientales.

\begin{abstract}
"The article discusses the need to undertake environmental studies, bringing in philosophical, historical, anthropological and political perspectives coming from the undeniable contribution of sociocultural studies. The first way to justify environmental studies is the understanding of environmental problems in each territory. The article talks about the narrow viewpoint coming from academic, natural sciences and social sciences, which interferes with conducting environmental studies from a cultural perspective. First, it discusses the origin of the term "environmental" in Latin American authors such as Daniel Vidart and Augusto Angel Maya. It then emphasizes different ways of approaching environmental studies: from single disciplinary studies to interdisciplinary studies, environmental knowledge and philosophy, social resistance, and indiscipline studies, ends up emphasizing the principal characteristics and the educational role which should be achieved in higher education on the environmental educational process, with the interdisciplinary exercise of environmental studies in national, regional and local areas. These last ideas are discussed in parts three and four.
\end{abstract}

Keywords: Sociocultural Studies, Enviromental Problems, Territory, Interdisciplinary, Enviroment Sciences. 


\section{INTRODUCCIÓN}

La discusión ambiental en Colombia se presenta en diferentes escenarios de carácter político, institucional, económico, social y en el ámbito académico. Sin embargo, la manera de asumir tanto sus discursos como sus prácticas suelen excluir,en vez de incluir, las diferentes miradas según los intereses de los agentes sociales institucionales, comunitarios, de los sectores productivos, los docentes como los investigadores. De este modo, existen quienes no pueden entender el ambiente más allá de su sentido ecológico, su actividad económica, como una metáfora relacionada con el sustento de vida e, incluso, como objeto de conocimiento. El presente artículo, al reconocer la base del ambiente en la relación sociedad naturaleza, exige una mirada complementaria tanto de los conocimientos como desde la práctica social frente a las problemáticas ambientales del territorio donde se dirime, día a día, un modelo sustentable de desarrollo.

En primer lugar se pregunta sobre el origen del concepto ambiente recalcando con autores como Daniel Vidart y Augusto Ángel Maya su triple dimensión: natural, humana y humanizada. En segundo lugar, desea evidenciar las diferentes modalidades de conocimiento que permiten el acceso a su estudio desde las ciencias ambientales donde se enfatiza el valor de los estudios ambientales como la manera idónea de construir la investigación interdisciplinaria, a partir del reconocimiento y comprensión de las problemáticas ambientales territoriales.

Seguidamente el artículo enfatiza en algunas propiedades de los estudios ambientales en las que se asumen, directamente, las necesidades socialmente sentidas de una problemática ambiental de la que han de surgir la selección de las disciplinas, saberes y metodologías que intentan ofrecer alternativas de solución a la disrupción entre la sociedad y la naturaleza en cada territorio. Finalmente, concluye con resaltar el valor de los estudios socioculturales para vincular las relaciones entre los presupuestos epistemológicos y la práctica institucional al centrar diversos campos de aplicación ambiental, en el reconocimiento de los aspectos estructurales que desde el ámbito biofísico, histórico, político y económico, sitúan la cultura como dimensión mediadora en la relación entre la sociedad con la naturaleza.

Una exhortación al papel de las universidades frente a un posible nuevo escenario posconflicto para Colombia, como eslabón entre la academia y las problemáticas ambientales del territorio, cierra el propósito general de la exposición.
I. El Germen de las Discusiones Ambientales: La Relación Naturaleza Sociedad

Daniel Vidart (1988) anota la confusión que contienen las consideraciones clásicas y contemporáneas sobre el ambiente. El ambiente, a diferencia de la expresión medio ambiente, no se podría restringir a un plano eminentemente de orden natural; pues, es necesario tener en cuenta, como su segunda dimensión, tanto la realidad cultural creada por el hombre como, a la vez, la realidad científico-tecnológica que ha conformado una tercera naturaleza ya no sólo en términos simbólicos sino, además, en sentido material. En otras palabras, el concepto de ambiente se circunscribe a la relación entre la dimensión natural, humana y humanizada.

La relación naturaleza y sociedad para Augusto Ángel Maya (1998) la equipara a la interacción ecosistema y cultura. Así como Daniel Vidart había escrito un libro sobre "La Filosofía Ambiental", Ángel Maya compendiará en tres cuadernos sobre ecosistema y cultura -“La Trama de la Vida" (1993), “La Tierra Herida" (1995) y “El Retorno a la Tierra"(1998)- la base ambiental sostenida en la relación entre la sociedad y la naturaleza:

"En el primer cuaderno explicamos el significado que le queremos dar al término ecosistema, reduciéndolo a la acepción acogida por los primeros ecólogos. Según esta definición el ecosistema es el conjunto de principios básicos, leyes o estrategias que rigen los sistemas vivos"(Ángel Maya 1993)

"En el segundo cuaderno describimos brevemente algunos de los impactos ambientales ocasionados por la actividad humana. Allí quedaron en suspenso, sin embargo, muchas ambigüedades. ¿A qué se debe el desorden que introduce el hombre en la trama de la vida?"(Ángel Maya 1995).

"El propósito principal del presente ensayo es, sin embargo, acercarnos a un análisis del sistema cultural desde la perspectiva ambiental. Cuando se plantea que la interferencia entre ecosistema y cultura es inevitable, se quiere dar a entender que el problema ambiental no depende del estilo peculiar del desarrollo moderno, sino que está implícito en cualquier sistema cultural histórico. (Ángel Maya 1998)

Tanto en Daniel Vidart como en Augusto Ángel Maya queda clara la existencia de un término medio común en la relación entre Naturaleza y Sociedad: La Cultura.

De la amplia gama de representaciones y prácticas, cada sociedad legitima una parte pequeña de estas: no sólo para 
posibilitar las relaciones entre sus áreas culturales (el arte, la religión, la ciencia, la economía, la política etc.) sino, también, para delinear sus propias manifestaciones simbólicas. Desde esta perspectiva, las sociedades han construido, merced a aquel sistema de símbolos elegidos, una cultura determinada a través de su desarrollo histórico. La cultura se convierte en aquel tejido o en aquella lente que permite comprender el mundo exterior $\mathrm{y}$, a la vez, como la principal estrategia humana para apropiar su entorno de acuerdo a sus deseos y necesidades.

De esta manera, la cultura, ha entrado a determinar el papel y la importancia de la naturaleza (Descolá 2012); por esta razón, es imposible pensar en una romántica relación armoniosa puesto que, contadas veces, de acuerdo a las necesidades e intereses de esta sociedad se llega a reducir el valor propio del horizonte biótico y abiótico del orden natural. Por lo tanto, el rol que cumplen las comunidades con sus respectivos sistemas de símbolos, prácticas y desarrollo científico tecnológico, incide en la configuración de los diferentes ambientes territoriales en cada nueva fase de la evolución histórica; en suma, no puede entenderse la transformación de los ecosistemas sólo circunscrita a explicaciones surgidas de procesos naturales.

La Cultura entre una de sus múltiples acepciones se podría entrar a definir, entonces, como una estrategia humana de adaptación tanto social como natural; posición a la que se adhiere Ángel Maya (1991). Una tarea irremplazable para develar las diversas reducciones del mundo natural por causa de las diferentes culturas justifica, por ejemplo, paradigmas de investigación adscritos a la ecología histórica (López 2008) y la historia ambiental (Gallini 2005).

El mundo occidental sería analizado entonces como el único sistema de valores y de símbolos que ha creado una historia universal de la cultura (Romero 1953). La época Moderna consolidó un pretendido sentido de unidad y homogeneidad al instaurar un concepto de progreso y desarrollo. La dicotomía existente entre naturaleza y sociedad en la época moderna, cuando el hombre se abroga el derecho de colocarse por encima de la misma, vino acompañada de otra dicotomía: la contraposición existente entre las ciencias naturales y las ciencias del espíritu.

Esta pugna ha ocasionado, por lo tanto, dos formas de reduccionismo: por un lado, las ciencias naturales, ante todo la física y la biología, han querido determinar las reglas del juego de las nacientes ciencias sociales; pero, por otro lado, las ciencias del espíritu no han podido encontrar una metodología adecuada para el estudio de sus difíciles objetos de conocimiento. En el primer caso, a las ciencias naturales les sobra el hombre $\mathrm{y}$, para el segundo caso, las ciencias sociales tratan al hombre más allá de sus consideraciones naturales.

Intentos como: el "Determinismo Ambiental" y el "Posibilismo", la "Ecología Humana" o el "Materialismo Cultural", la "Ecología Cultural" o la "Ecología Política", citados por Lemkov (2002) han tratado de salvar las barreras entre estas dos tendencias antagónicas, desde el marco epistemológico no sólo de la filosofía sino, además, de la geografía, la historia, la antropología, la sociología e incluso la biología y la ecología; pero, aunque han ofrecido valiosos elementos de discusión y aportes metodológicos, no han podido solucionar el alejamiento de estos dos saberes, presupuesto esencial para la construcción de conocimiento interdisciplinario, en pos de un marco de referencia común para las ciencias ambientales.

Nuestra tarea será intentar reconocer algunas de las principales estrategias asumidas por investigadores, académicos y ambientalistas colombianos y latinoamericanos que intentan legitimar, la existencia de un nuevo campo de conocimiento llamado las ciencias ambientales, sobre la base de la relación cultura y ambiente.

\section{Diferentes Modalidades de Conocimiento para el Acceso a las Ciencias Ambientales}

Hablar de las ciencias ambientales en Colombia exige remitirse a una serie de tradiciones que, desde la participación y énfasis de diferentes agentes sociales, han intentado nombrar algunas modalidades de acceso a esta nueva área de conocimiento para el país (Sáenz 2007). Desde el aporte de las disciplinas, la multidisciplina, los estudios ambientales, la filosofía ambiental, el saber ambiental, y la "indisciplina"; se ha tratado de dar explicación a las relaciones entre la sociedad y la naturaleza, germen de toda discusión ambiental.

En primer lugar, no se puede negar la contribución de una serie de representantes de diferentes disciplinas quienes, gracias a introducir como variable o dimensión la temática ambiental, han posibilitado ampliar los halos de comprensión de diversas disciplinas. La economía ambiental, la geología ambiental, la historia ambiental, la química ambiental, la antropología ambiental, entre otras disciplinas, ayudan a reconocer la contribución de autores como Héctor Sejenovich, Michel Hermelin, Francisco González, por ejemplo, en la configuración de las ciencias ambientales.

En segunda medida, sectores relacionados con la gestión y la 
práctica ambiental han demostrado la necesidad de realizar diagnósticos ambientales integrales, estudios de impacto ambiental, propuestas de desarrollo rural y/o urbano, desde miradas multidisciplinarias. Estos proyectos e, incluso, investigaciones muestran la contribución de las ciencias naturales, las ciencias sociales y la ingeniería aplicada, en análisis más amplios de carácter ambiental.

La interdisciplinariedad que se intenta construir desde los referentes académicos de los estudios ambientales, también es una ventana de acceso para la construcción de las ciencias ambientales. Cuando una serie de especialistas de diferentes disciplinas se encuentran con desafíos ambientales en cada territorio deben, en lo posible, buscar la articulación de conocimientos que les exige la problemática ambiental a afrontar. Los estudios ambientales, como tercera modalidad de conocimiento y acceso, permiten entender por qué la investigación interdisciplinaria surge directamente de las problemáticas ambientales, y no obedece a unos conocimientos apriori definidos al margen de la experiencia real.

Profesores como Augusto Ángel Maya (1993), Enrique Leff (2008),Ana Patricia Noguera (2004),desde la lectura de filósofos de la antigüedad clásica, el mundo moderno y contemporáneo han ayudado a explicitar la pertinencia de diferentes epistemologías en la reflexión ambiental. Esta cuarta modalidad de conocimiento, ha aportado categorías epistemológicas como: el Núcleo de la Problemática Ambiental (Maya 1991), La Racionalidad Ambiental (Leff, 2008) la Producción Ecotecnológica (Leff, 2002), entre otros conceptos, y evidencian la importancia del pensamiento filosófico, la ética y la estética para la justificación académica de las ciencias ambientales.

Una quinta modalidad, con profundos arraigos en la tradición antropológica, es la noción del saber ambiental. Para Enrique Leff, el saber ambiental (2002) reivindica otros tipos de conocimientos, ajenos a la ciencia, que legitiman desde la cultura, las relaciones entre la sociedad y la naturaleza en contextos como nuestro continente latinoamericano. Las cosmovisiones de nuestros pueblos originarios pueden demostrar la sustentabilidad histórica de nuestras costumbres y prácticas, para ser tenidas en cuenta en los procesos tanto de innovación tecnológica como de rescate del patrimonio cultural ambiental.

En horizontes actuales, se podría adicionar como otra posible ventana de acceso a las ciencias ambientales, la resistencia social de nuestras comunidades desde los movimientos ambientales y el ambientalismo raizal (FalsBorda 2008).Una lectura comprometida con la naturaleza y con la vida en cada una de sus demandas ambientales ha hecho posible, en palabras de Arturo Escobar (1994), el valor conferido a las diferencias en el mundo del posdesarrollo.

Por último, y desde la trayectoria y experiencia del maestro Julio Carrizosa (2014), hemos de entender otra manera alternativa de acceder al conocimiento de las ciencias ambientales: el camino autodidacta emprendido por la indisciplina ambiental. Nuestros terruños no sólo han producido la riqueza y complejidad ambiental de los ecosistemas sino, además, hombres únicos que han enseñado por generaciones a profesionales y al colombiano del común, el valor de nuestro ambiente: Jorge el "Mono" Hernández, Aníbal Patiño, Gonzalo Palomino, Guillermo Castaño etc. Algunos desde el ambientalismo pero todos desde un proyecto de vida llamado el ambiente, se han convertido en verdaderos referentes de la investigación y comprensión de problemas que atañen directamente a las ciencias ambientales.

Las ciencias ambientales, entonces, desde este variopinto abanico, podrían comprenderse desde estas modalidades de conocimiento.

\section{Estudios Ambientales y Problemática Ambiental}

Como se anotaba en un párrafo anterior, los estudios ambientales permiten la comprensión interdisciplinaria de las problemáticas ambientales en los territorios concretos (Cubillos 2015). Si la definición de lo ambiental atañe a una relación entre la sociedad y la naturaleza, los estudios ambientales confrontan dicha relación teniendo en cuenta que las culturas pertenecientes a los diferentes agentes sociales presentes en los territorios, se convierten en el principal filtro para poder entender los aspectos simbólicos y prácticos de las relaciones ambientales.

Sin embargo, la historia del mundo occidental ha producido en la escala global, una disrupción en la relación existente entre los ecosistemas y las culturas, lo que en palabras de Augusto Ángel Maya (1991) se ha convertido en el Núcleo de la Problemática Ambiental cuando la especie humana pone en riesgo los límites naturales de la vida. $L a$ problemática ambiental, así entendida, exigiría indagar en cada territorio cómo se manifiesta esta ruptura entre la sociedad y la naturaleza, en procura de la comprensión cultural y la aplicación futura de propósitos de gestión ambiental, hacia la sostenibilidad y sustentabilidad de las futuras decisiones ambientales.

Esta ruptura sociedad - naturaleza se presencia, incluso, en nuestra cultura académica lo que puede distanciar los 
esfuerzos logrados por el pensamiento y filosofía ambiental de las actividades y preocupaciones propias de los conocimientos multi y disciplinarios. Ya en el año 2007 en el volumen editado por la Red de Formación Ambiental para Colombia y Colciencias sobre "Las Ciencias Ambientales como Nueva Área de Conocimiento para Colombia" (Saénz 2007) en mi artículo: "La Epistemología Ambiental: Reflexiones desde la impertinencia social anotaba lo siguiente:

"Cuando se escribe o se habla en términos epistemológicos se puede incurrir en dos graves extremos: supeditar cualquier tema de discusión científica al orden de la larga tradición y de los afectos filosóficos, o restringir los planteamientos al marco preestablecido de una determinada área de estudio puesta en cuestión.

Más que encuentros entre filósofos y científicos se presentan desencuentros entre quienes tienen una presumible visión integral de los planteamientos epistemológicos desde las escuelas filosóficas -con el gran agravante, muchas veces, de desconocer los procesos, técnicas e instrumentos de la investigación científica- o con aquellos que poseen el conocimiento inherente que le ofrecen sus disciplinas, sin contar con la formación global que le permita reflexionar más allá de las mismas.

Si a la epistemología también le interesa realizar un análisis de las connotaciones históricas, políticas, sociales y culturales sobre el origen y evolución de las ciencias (Thuillier1975) más que una mirada externalista, visión filosófica, o internalista, epistemología de la ciencia, se debe propender por una vía intermedia que reconociendo el aporte innegable de las escuelas, pensadores y debates filosóficos se cuide, por una parte, de sentenciar desde allí los límites y alcances de todo conocimiento científico; pero que, por otra parte, se abstenga de incidir en procesos endógenos interesados en hablar de las ciencias desde las ciencias mismas.

En ambos casos la sociedad es sacrificada por entrar en una discusión en la que ni filósofos ni científicos se ponen de acuerdo; de entrada ambos deberían preguntarse: Cómo encausar el deber ser de estas ciencias de acuerdo a las demandas y necesidades más sentidas de los diferentes agentes sociales habitantes de un determinado territorio?"

Pensar las relaciones actuales entre la sociedad y la naturaleza, desde la categoría de la problemática ambiental del territorio, compromete a los investigadores con estudios de carácter interdisciplinario a partir de los contextos propios donde se manifiestan los problemas ambientales. Dichos problemas ambientales no se pueden reconocer desde un punto de vista aislado o atomizado (García 2006), pues la categoría superior de la problemática ambiental exige, por una parte, el concurso de diversas disciplinas y saberes que, de manera articulada, tratan de ofrecer posibles alternativas de solución a las situaciones ambientales a partir, por otra parte, de comprender y explicar las causas de fondo de la problemática ambiental en cuestión.

Cada problemática ambiental exige unos conocimientos, saberes, métodos y la participación de agentes sociales que no se pueden definir sin conocer cómo se desenvuelven, influencian y son influenciados por el ambiente. Los aspectos culturales son prerrequisito ineludible para explicar las razones históricas, sociales y políticas de cada problemática ambiental (Cubillos 2009).

En otras palabras, la exposición de los problemas ambientales específicos y aislados que tradicionalmente señalan el deterioro ambiental (contaminación de fuentes hídricas, disposición inadecuada de residuos sólidos, falta de espacios públicos, pérdida de cobertura vegetal, presencia de amenazas naturales y antrópicas, etc.) sugieren causas estructurales que, desde el corazón de la cultura, dan cuenta de la desarticulación entre la sociedad con la naturaleza, en los territorios concretos donde se lleva a cabo un proceso de investigación interdisciplinaria.

\section{Conclusiones: Los Estudios Socioculturales y la Problemática Ambiental del Desarrollo}

Aunada a la discusión académica existente entre la filosofía ambiental y los científicos ambientales, se puede reconocer un segundo conflicto entre la práctica ambiental de las instituciones y las recomendaciones de la epistemología ambiental. A menudo los fines últimos de los estudios ambientales y las actuaciones institucionales frente a la gestión ambiental del desarrollo se presentan como dos polos irreconciliables: en una arista, la epistemología ambiental aspira a identificar presupuestos científicos "deseables" (la visión sistémica, la complejidad, la inter y transdisciplinariedad etc.) allende a las problemáticas ambientales concretas $\mathrm{y}$, en la otra arista, la gestión ambiental privilegia una actuación práctica donde las normas, la economía y los procedimientos técnicos imperan sobre cualquier discusión académica.

Desde un punto de vista que privilegia la comprensión, evaluación y posible intervención sobre crisis ambientales concretas, ambas perspectivas surgen de espacios equívocos: de unas pretendidas ciencias ambientales existentes y regladas por principios "científicos" integrales, o desde unos aspectos formales preexistentes sean institucionales, técnicos o económicos que rigen la práctica ambiental institucional. En ambos casos, los aspectos socioculturales de las realidades ambientales son dejados de lado; al confinar las discusiones y estudios históricos, antropológicos y políticos de la temática ambiental, a planteamientos teóricos 
generales o al reducir los aspectos sociales de la gestión ambiental a la participación y al aval comunitario, produciendo, al final, una visión aséptica de las consideraciones culturales y de las relaciones de poder en la política ambiental.

Cuando temáticas tan relevantes como el ordenamiento ambiental del territorio, la planificación del desarrollo, el cambio climático, la gestión del riesgo, el manejo de la conservación, entre otros ejemplos, se atienden enfatizando la construcción cultural del territorio-más allá del espacio físico-; los procesos endógenos y alternativos de y al desarrollo- allende al reduccionismo económico-; la configuración histórica de los procesos de vulnerabilidad biofísica y sociocultural de nuestros ecosistemas y sociedades- sin restringir los desastres a razones eminentemente naturales-; políticas de manejo de parques naturales con la participación comunitaria,-sin expulsar la gente de su tierra-, se está realmente confiando en que la orientación integral, reguladora y axiológica de la cultura debe sustentar con el tiempo, el ejercicio de la toma de decisiones ambientales.

La política y la planeación ambiental, por lo tanto, tendrían que entrar a considerar los aspectos culturales en sus indicadores de gestión, recordando siempre que la viabilidad de cualquier plan, programa o proyecto no está garantizada por la eficiencia formal en la búsqueda de sus objetivos, sino, principalmente, en los procesos de sustentabilidad social que hagan posible las articulaciones entre las organizaciones, las sociedades, los sectores productivos y la academia.

Una nueva locomotora basada en el desarrollo rural y el crecimiento verde aparece en el nuevo plan de desarrollo "Todos por un Nuevo País" (2015) soportado en los principios de la Paz, la Equidad y la Educación. Las equivocaciones de las anteriores cinco locomotoras, sobre todo la locomotora minero energética, no pueden repetirse, $\mathrm{y}$, en buena parte, estas equivocaciones estuvieron amparadas por el desconocimiento de las bases ambientales y culturales de estas "pretendidas" actividades económicas; Julio Fierro (2012), Luis Jorge Garay (2012), Álvaro Ponce (2013), Aurelio Suarez (2013), Carolina Arias(2013), entre otros autores, han demostrado los conflictos ambientales derivados de la incomprensión de las políticas ambientales mineras frente a los beneficios políticos, económicos e incluso legales que han tenido otro tipo de minería foránea.

Las problemáticas y oportunidades ambientales del país parecieran hoy querer leerse desde un nuevo y añorado escenario de posconflicto (mejor llamarlo posacuerdo); la relación naturaleza y sociedad necesita mutuamente reconocerse sobre todo en las instancias competentes de la gestión ambiental tanto en el orden nacional como en los ámbitos locales y regionales. La Educación Superior, en suma, está llamada a convertirse en esa eslabón responsable con la mirada integral de los territorios donde se unan no solamente las ciencias y saberes pertinentes sino, además y principalmente, los diferentes agentes sociales comprometidos a brindar posibles alternativas de solución, gracias a la garantía que ofrecen los estudios ambientales.

\section{REFERENCIAS}

ARIAS, C. 2013. ¿Neoestractivismo Desarrollo Local? Conflictos Territoriales y Patrimoniales en el Pueblo Minero de Marmato, Colombia. Tesis de Maestría en Gestión del Patrimonio y Desarrollo Territorial. Universidad Mayor de San Simón, Cochabamba, Bolivia. 196 pp.

CARRIZOSA, J. 2014. Colombia Compleja. Jardín Botánico de Bogotá, Colombia. 295 pp.

CUBILLOS, L. 2009. La Gestión Cultural Ambiental frente al Desafió de las Problemáticas Ambientales Reales. En: López, C. (compilador) Diálogos entre Saberes Ciencias e Ideologías en Torno a lo Ambiental. Editorial Publiprint, Pereira, Colombia.213 pp.

CUBILLOS, L. 2007. Epistemología de las Ciencias Ambientales: Reflexiones desde la "impertinencia Social". En: Sáenz, O. (Compilador). Las Ciencias Ambientales como Nueva Área de conocimiento. COLCIENCIAS. Bogotá.220 pp.

CUBILLOS, L. 2010. Pretensiones Académicas frente al Proceso Fundacional de una Nueva Área de Conocimiento para Colombia. En: Rodríguez, D. y García, A. (Editores.). Cátedra Ambiental: Memorias 2006 - 2009. Un Espacio de Reflexión para la sustentabilidad. Universidad Tecnológica de Pereira. 224 pp.

CUBILlOS, L. 2015. Razones Académicas para la Justificación de las Prácticas Ambientales Interdisciplinarias. En: Arias Carolina (compiladora) La Resignificación de la Cuenca del Consota, Risaralda Colombia. Talleres Publiprint, Pereira, Colombia. 335 pp.

DESCOLÁ, P. 2012. Más Allá de Naturaleza y Cultura, Amorrortu, Buenos Aires, Argentina. Postergraph S.A. Dosquebradas, Colombia.624 pp.

ESCOBAR, A. 1994. La Invención del Tercer Mundo. Editorial Norma, Bogotá, Colombia. 475 pp. 
FALS, O. 2008. Hacia un Socialismo Raizal y Otros Escritos. Ediciones Desde Abajo. Bogotá, Colombia. 120 pp.

FIERRO, J. 2012. Políticas Mineras en Colombia. De Aquí y Ahora. Digiprinto Editores, Bogotá, Colombia. 259 pp.

GALLINI, S.2005.Invitación a la historia ambiental.En: Revista Tareas Nro. 120: Historia ambiental Latinoamericana. Mayo-Agosto 2005. CELA, Centro de Estudios Latinoamericanos "Justo Arosemena", ciudad de Panamá, Panamá. 5-28 pp.

GARAY, L. 2013. Minería en Colombia: Derecho, Políticas Públicas y Gobernanza. Contraloría General de la República. Bogotá, Colombia. 211 pp.

GARCÍA, R. 2006. Sistemas Complejos. Gedisa, Barcelona, España. 202 pp.

LEFF, E. 1998/2002. Saber ambiental. Racionalidad, sustentabilidad, complejidad, poder: Siglo XXI Editores. México. 414 pp.

LEFF, E. 2004. Racionalidad ambiental. La apropiación social de la naturaleza. Siglo XXI Editores. Ciudad de México. México. 507 pp.

LEFF, E. 2008. Discursos Sustentables. Siglo XXI Editores. Ciudad de México, México. 271 pp.

LEMKOW, L. 2002. Sociología Ambiental. Pensamiento Socioambiental y Ecología Social del Riesgo. Icaria, Barcelona, España. 231 pp.

López, C. (compilador). 2009. Diálogos entre Saberes Ciencias e Ideologías en Torno a lo Ambiental. Editorial Publiprint, Pereira, Colombia. 213 pp.

MAYA, A. 1993. La Trama de la Vida: Bases ecológicas del pensamiento ambiental. CUADERNOS AMBIENTALES, serie ecosistema y cultura. No 1. Ministerio de Educación Nacional, IDEA Universidad Nacional de Colombia. 75 pp.

MAYA, A. 1995. La Tierra Herida: Las transformaciones tecnológicas del ecosistema. CUADERNOS AMBIENTALES, serie documentos especiales. No 2. Ministerio de Educación Nacional, IDEA Universidad Nacional de Colombia. 82 pp.
MAYA, A. 1998. El Retorno a la Tierra: Introducción a un Método de Interpretación ambiental. CUADERNOS AMBIENTALES, serie documentos especiales. No 3. Ministerio de Educación Nacional, IDEA Universidad Nacional de Colombia. 75 pp.

NOGUERA, A. 2004. El Reencantamiento del Mundo. PNUMA - UNAL. Talleres de Gráficas JES, Manizales. 206 pp.

PONCE, Á. 2012. ¿Cuál Locomotora? El Desalentador Panorama de la Minería en Colombia. Nomos Impresoras. Bogotá, Colombia. 213 pp.

PRESIDENCIA DE LA REPÚBLICA DE COLOMBIA. 2015. Plan de Desarrollo: Todos por un Nuevo País: Paz, Equidad y Educación. Versión Preliminar de Discusión. DNP Bogotá, Colombia

ROMERO, J. 1953. La Cultura Occidental. Editorial Siglo XXI. Ciudad de México, México. 95 pp.

SUAREZ, Aurelio.2013. La Minería Colonial del Siglo XXI. No Todo lo que Brilla es Oro. Ediciones Aurora. Bogotá, Colombia.154 pp.

VIDART, D.1984. Filosofía Ambiental. Editorial Nueva América. Bogotá, Colombia. 542 pp. 